\title{
A Simplified Hybrid Method for Calculating the Frequency-dependent Inductances of Transmission Lines with Rectangular Cross Section
}

\author{
Shuzhou Fang Xiaobo Tang Zeyi Wang Xianlong Hong \\ Dept. of Computer Science \& Technology, \\ Tsinghua University, \\ Beijing 100084 \\ Tel: +86-10-62785564 \\ Fax:+86-10-62781489 \\ Email: fsz@263.net
}

\begin{abstract}
This paper describes a simplified hybrid method to calculate the frequency-dependent inductances of transmission lines, which combines cross-section coupled circuit method with boundary element method (BEM) just solving Laplace's equation. The coupled circuit method is used to calculate inductance in low frequency range; The BEM builds the capacitance matrix, then we invert this matrix (omit the row and column relate to ground plane), we can obtain inductance relating to the high frequency at which the current only distributes on surfaces of conductor. A cubic spline interpolation between inductance at low and very high frequency gives a good result over entire frequency range. Comparing this method with previous hybrid method [3], it avoids solving Helmholtz's equation and obtains the capacitance matrix at the same time, greatly reducing the total burden of calculation. Numerical tests show it is applicable and efficient.
\end{abstract}

\section{INTRODUCTION}

It is necessary to calculate frequency-dependent inductance when the density and speed of integrated circuits increases rapidly. But today there isn't a single method can be applicable in entire frequency range.

When the frequency is low, in cross-section coupled circuit method [1], the conductor cross section is divided into patches, assuming the distribution of current is uniform. A patch relates to a filament, and all filaments consist an impedance network, complied with Kirchoff's laws. The resistance and mutual inductance between the filaments are calculated, and a matrix is inverted to give the overall resistance and inductance of the conductors. This method is not suitable for high frequency because it can't model singularities of the current density on the cross section.

When the frequency is high, the boundary integral equation method can be adopted [2]. The inside regions of conductors are governed by Helmholtz's equation while outside region are governed by Laplace's equation. The density of current and its normal derivative on boundary are taken as the unknowns and substituted into the boundary conditions, and an integral equation is obtained. It can be solved by BEM. The inductance and resistance are expressed in terms of boundary current density and its boundary normal

This project is supported by 973 National Key Project on Foundational Research derivative. In low frequency range, the current density normal derivative is small, therefore the numerical error is high.

In [3], the hybrid method combines above two methods to calculate inductance and resistance in low and high frequencies respectively, then an interpolation between these results is made to obtain inductance and resistance in entire frequency range.

In this paper, we suggest a simplified hybrid method, which invert capacitance matrix to obtain high frequency inductance to perform interpolation, instead of solving Helmholtz's equation. Because the inductance curve in high end of frequency range approaches to a constant, we think just two points are sufficient to make an accurate cubic spline interpolation, combining points calculate by coupled circuit method in low frequency range.

The calculation needed by our simplified hybrid method is much less than that of the hybrid method in [3]. Our method only needs to solve just one Laplace's equation to obtain capacitance matrix and then invert it to inductance matrix. But the hybrid method must solve Helmholtz's and Laplace's equations coupled on conductor surfaces to get high frequency inductance. It also needs to solve another Laplace's equation to obtain capacitance matrix separately. To solve Helmholtz's and Laplace's coupled equations, each point on conductor surfaces has two unknowns: $J$ and $\partial J / \partial n$ (current density, its boundary normal derivative respectively), but to solve Laplace's equation there is only one unknown: $\partial u / \partial n$ or $u$ (electrical potential, its boundary normal derivative respectively), and the integral kernel of Laplace's equation is much simple than that of Helmholtz's equation. Because the number of conductors is much less than that of unknowns, the calculation of inverting capacitance matrix in our method can be negligible.

\section{COUPLED CiRCUit METHOD IN LOW FREQUENCY RANGE}

In low frequencies, there are multiple transmission lines (wires) over a common return path or "ground plane." The 
resistance and inductance matrices $R$ and $L$ are defined by [3]

$$
d V / d z=(i \omega L-R) \cdot I
$$

$V$ is the column vector of the voltage differences between the wires and ground plane, $z$ is the current direction, and $I$ is the column vector of currents in the wires. The cross section of each conductor (signal wire or ground plane) is divided into triangular patches and one patch of the ground plane is chosen to be the voltage reference. The current density is assumed uniform on each patch. Each patch relates to a filament, the voltage vector $v$ and current vector $i$ corresponding to all the filaments have the relationship

$$
d v / d z=(i \omega l-r) \cdot i
$$

Where $r$ is resistance matrix and $l$ is inductance matrix of filaments. A component of $r$ is

$$
\begin{aligned}
& r_{j k, j k}=\frac{1}{\sigma A_{j k}}+\frac{1}{\sigma A_{00}} \\
& r_{j k, m n}=\frac{1}{\sigma A_{00}}, \quad j \neq m, k \neq n
\end{aligned}
$$

$r_{j k, m n}$ is the unit length resistance of $k$ patch on $j$ wire cause by $n$ patch on $m$ wire, $\sigma$ is conductivity of the wire, $A$ is area of the patch, and patch 0 on wire 0 is the voltage reference patch on ground plane. Following variables have same subscript denotation. The component of $l$ is

$$
l_{j k, m n}=l_{j k, m n}^{(p)}-l_{j k, 00}^{(p)}-l_{00, m n}^{(p)}+l_{00,00}^{(p)}
$$

Where partial inductances with $(p)$ superscript

$$
l_{j k, m n}^{(p)}=-\frac{\mu}{4 \pi A_{j k} A_{m n}} \iint d S_{j k} \iint d S_{m n}^{\prime} \cdot \ln \left[\left(x-x^{\prime}\right)^{2}+\left(y-y^{\prime}\right)^{2}\right](5)
$$

Where $\mu$ is permeability of the wire, $x$ and $y$ are coordinates on patch $j k$, and $x^{\prime}$ and $y^{\prime}$ are coordinates on patch $m n$. The integral has closed form [3]. Define $z=-[i \omega l-r]$, $y=z^{-1}$, we have

$$
I_{j k}=-\sum_{m=0}^{N} \sum_{n=1}^{N_{m}} y_{j k, m n} \frac{d V_{m n}}{d z}
$$

Where $N$ is the number of wires and $N_{m}$ is the number of patches on wire $m$. Because of quasi-TEM assumption which means that there aren't transverse currents, the voltage of each patch in a wire is the same, and the total current in each wire is the sum of the currents in its patches. Apply these conditions to $v$ and $i$, gives

$$
I_{j}=-\sum_{m=1}^{N} Y_{j m} \frac{d V_{m}}{d z}
$$

Where $\quad Y_{j m}=\sum_{k=1}^{N_{j}} \sum_{n=1}^{N_{m}} y_{j k, m n}$

Invert $Y$ gives

$$
Y^{-1}=R-i \omega L
$$

Thus we get frequency dependent resistance and inductance.

The cross section coupled circuit method is only suitable in low frequencies. When frequency increases, the current concentrates on the edges, then the dimension of the patch should be consistent with skin depth, so the number of patches increases rapidly, and the distribution of patches should also be adjusted according to the frequency.

\section{CAlculation of CAPACitAnCE By BEM}

The parasitic capacitances of the transmission lines over infinitive ground plane in single media can be calculated by solving the Laplace's equation using BEM [5]. The equation and boundary conditions are:

$$
\left\{\begin{array}{l}
\nabla^{2} u=0 \quad \text { in } \Omega \\
u=u_{0} \quad \text { on } \Gamma_{u} \\
q=\frac{\partial u}{\partial n}=q_{0}=0 \text { on } \Gamma_{q}
\end{array}\right.
$$

The boundary of domain $\Omega$ is $\Gamma=\Gamma_{u}+\Gamma_{q}, \Gamma_{u}$ is forced boundary, where the potential $u$ is known constant decided by bias voltage. $\Gamma_{q}$ is free boundary where $\partial u / \partial n$ is zero, $n$ is unit normal vector of $\Gamma$.

With the fundamental solution $u^{*}$ as the weighting function, the Laplace's equation and its mixed boundary conditions are transformed into the boundary integral equation using the weighted residual method

$$
c_{s} u_{s}+\int_{\Gamma} q^{*} u d \Gamma=\int_{\Gamma} q u^{*} d \Gamma
$$

Where $u_{s}$ is the potential of point $s, c_{s}$ is a constant dependent on boundary smoothness near point $s$. In 2D situation, the fundamental solution of the domain which has an infinitive ground plane is

$$
u^{*}=\frac{1}{2 \pi \varepsilon} \ln \frac{\left|\rho-\hat{\rho}^{\prime}\right|}{\left|\rho-\rho^{\prime}\right|}
$$

Where $\varepsilon$ is permittivity, $\rho$ is radius vector of $s, \rho, \hat{\rho}$ are radius vectors of source point and its image about ground plane. After discretizing the boundary $\Gamma$ and substituting boundary conditions into discretized integral equation (10), a system of linear equations is obtained: 


$$
A x=f
$$

Where column vector $x=\left[x_{u}, x_{q}\right]^{T}, x_{u}$ is $N_{u}$ nodal normal field $q$ on $\Gamma_{u}, \quad x_{q}$ is $N_{q}$ nodal potential $u$ on $\Gamma_{q}$, and total number of unknowns is $N=N_{u}+N_{q}$. Only one conductor is given 1 volt bias voltage at each time, so the most elements of $f$ are zero except those on the 1 volt conductor. After the system of linear equations (12) is solved, we get one column of capacitance matrix. Setting 1-volt voltage to rest of conductors and calculating with same procedure, the whole capacitance matrix $C$ is resulted.

\section{INTERPOLATION METHOD}

In [4] the authors have proved following formula when the current distributes only on conductor surfaces.

$$
L=\mu \varepsilon[C]^{-1}
$$

$L, C$ are inductance and capacitance matrices. $\mu, \varepsilon$ are permeability and permittivity. The skin depth of rectangular cross section conductor is

$$
\delta=\sqrt{1 /(\pi f \mu \sigma)}
$$

Where $f$ is frequency $(\mathrm{Hz}), \sigma$ is conductivity.

Assume the mean dimension of the cross section of wires is $a$. When $2 \delta>>a$, the skin effect can be ignored, current distributes evenly; when $2 \delta / a \rightarrow 0$, this means the current concentrates on the conductor surfaces, and we can relate the frequency causing this singularity to the inductance calculated by inverting capacitance matrix.

Define $p=2 \delta / a$, from (14) we have

$$
f=\frac{4}{\pi \mu \sigma p^{2} a^{2}}
$$

Our interpolation method has following steps:

1. Choose $p=p_{\text {low }}=4 \sim 10$, calculate $f=f_{\text {low }}$

2 . In the frequency range of $0 \sim f_{\text {low }}$, calculate $n$ interpolation points $\left(f_{i}, L_{i}\right) i=1,2, \ldots n$ by coupled circuit method.

3. Choose $p=p_{\text {high }}=0.05 \sim 0.01$, calculate $f_{\text {high }}=f$.

4. If the inductance of a conductor calculate by inverting capacitance matrix is denoted as $L_{C}$, choose $f_{\text {high }}>f_{\text {high }}$, last two interpolation points are $\left(f_{\text {high }}, L_{c}\right)$ and $\left(f_{\text {high }}, L_{c}\right)$.

5. Make a cubic spline interpolation between these $n+2$ points, obtain the inductance curve over the entire frequency range.

\section{RESULTS}

Next we compare our method with the hybrid method in [3]. We will also show that the accuracy of our method is not sensitive to the variation of $p$.

The case is three rectangular conductors over ground plane, denoting as conductor 1,2 , and 3 . They have same crosssection, evenly distribute. The conductivity of all conductors is $\sigma=5.81 \times 10^{7}(\Omega / m)^{-1}$, and they are all in free space. The configuration is shown in Fig. 1.

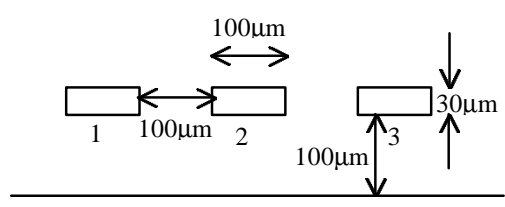

Fig. 1. Three rectangular wires over a ground plane.

The capacitance matrix calculated by solving Laplace's equation using BEM is showed in Table I. The inductances calculated by inverting capacitance matrix (ground plane is omitted) are showed in Table II. Two of inductances calculated from capacitance matrix are $L_{11}=376.3(\mathrm{nH} / \mathrm{m}), L_{12}=99.44(\mathrm{nH} / \mathrm{m})$.

In [3], this case is most complex example, but they didn't give the numbers of the inductances. We use the fig. 10 in [3] to compare the results of our method and their hybrid method. We choose $n=3$. When we choose $p_{\text {low }}=4$, $p_{\text {high }}=0.05$, we can obtain $\lg \left(f_{\text {low }}\right)=5.4$ and $\lg \left(f_{\text {high }}\right)=9.2$, the result is showed in Fig. 2; When we choose $p_{\text {low }}=10, p_{\text {high }}$ $=0.01$, we can obtain $\lg \left(f_{\text {low }}\right)=4.6$ and $\lg \left(f_{\text {high }}\right)=10.6$, the result is showed in Fig 3 .

It can be seen that the curves are nearly overlapped. It also can be seen that our interpolation method is not sensitive to the change of $p$ in a quite wide range. It means the choice of the $p$ is not a difficult task. The calculations show that the accuracy will be improved, if the $p_{\text {low }}$ is small and $p_{\text {high }}$ is large, while the skin effect can be still ignored at frequency smaller than $f_{\text {low }}$ and well developed at frequency higher than $f_{\text {high }}$. It is because last two interpolation points will be closer to first $n$ points. The results show that $n$ could be small, only $n=3$ can gives a good interpolation result.

TABLE I

CAPACITANCE MATRIX

\begin{tabular}{|c|c|c|c|c|}
\hline Unit $(\mathrm{pF} / \mathrm{m})$ & 1 & 2 & 3 & Ground \\
\hline 1 & 31.8655 & -8.2950 & -1.0431 & -22.5273 \\
\hline 2 & -8.2963 & 34.5809 & -8.2945 & -17.9898 \\
\hline 3 & -1.0419 & -8.2959 & 31.8680 & -22.5300 \\
\hline Ground & -22.5273 & -17.9900 & -22.5304 & 63.0471 \\
\hline
\end{tabular}

TABLE II

THE INDUCTANCE MATRIX BY INVERTING CAPACITANCE MATRIX

\begin{tabular}{|c|c|c|c|}
\hline $\operatorname{unit}(\mu \mathrm{H} / \mathrm{m})$ & 1 & 2 & 3 \\
\hline 1 & 0.376306 & 0.099429 & 0.038197 \\
\hline 2 & 0.099439 & 0.369455 & 0.099415 \\
\hline 3 & 0.038189 & 0.099427 & 0.376272 \\
\hline
\end{tabular}




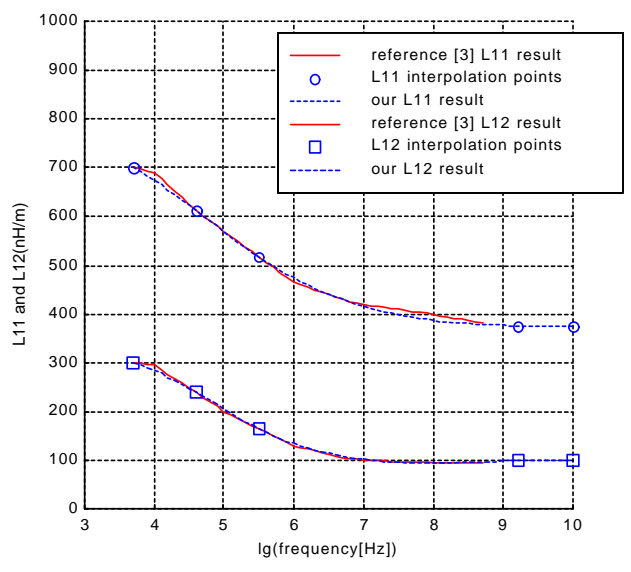

Fig. 2. Results when $p_{\text {low }}=4, p_{\text {high }}=0.05$

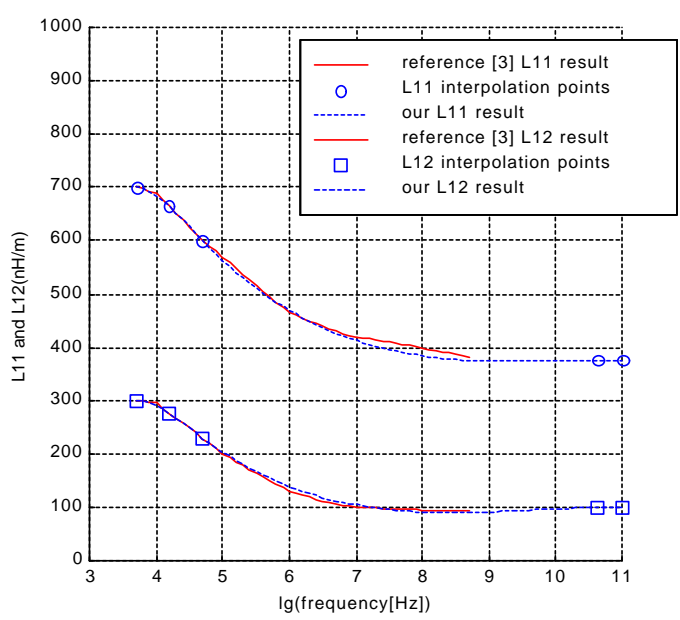

Fig. 3. Results when $p_{\text {low }}=10, p_{\text {high }}=0.01$
Because we can't access to the program used in [3], the exact speed improvement of our method is not available. But we solve Laplace's equation instead solve Helmholtz's equation by BEM, and we obtain capacitance matrix at the same time, all of these simplicity in algorithm can guarantee significant reduction of calculation.

\section{CONCLUSIONS}

A simplified hybrid method has been developed to calculate inductance in entire frequency range and obtain capacitance matrix at the same time. It combines the coupled circuit method to calculate low frequencies inductance and BEM to obtain capacitance matrix by solving just Laplace's equation. After inverting capacitance matrix to obtain the high frequency inductances, a cubic spline interpolation is make between these result to obtain the entire frequency range inductance. This method avoids solving Helmholtz's equation, greatly reducing the burden of calculation. Numerical results show it is applicable and efficient.

\section{REFERENCES}

[1] W. T. Weeks, L. L. Wu, M. F. McAllister, and A. Singh, "Resistive and inductive skin effect in rectangular conductors," IBM J. Res. Develop., Vol. 23, no. 6, pp. 652-660, Nov. 1979.

[2] R. B. Wu and J. C. Yang, "Boundary integral equation formulation of skin effect problems in multiconductor transmission lines," IEEE Trans. Magn., vol. MAG-25, pp. 3013-3015, July 1989.

[3] Michael J. Tsuk and Jin Au Kong, "A hybrid method for the calculation of the resistance and inductance of transmission lines with arbitrary cross sections," IEEE Trans. on MTT., Vol. 39, No. 8, pp. 1338-1347, Aug. 1991.

[4] Cao Wei, R. F. Harrington, J. R. Mautz, and T. K. Sarkar, "Multiconductor transmission lines in multilayered dielectric media," IEEE Trans. on MTT, Vol. 32, No.4, April 1984.

[5] Zeyi Wang and Qiming Wu, "A two-dimensional resistance simulator using the boundary element method," IEEE Trans. on CAD, Vol. 11, No. 4, April 1992. 